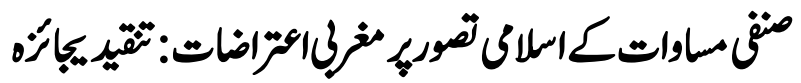

\section{Western Objections to the Islamic Concept of Gender Equality: A Critical Review}

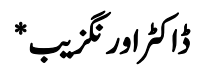

\begin{abstract}
Over the past several years, despite the constant evolution of the legislation the problem of gender equality in the West has been steadily increasing. What is the reason why there is no significant progress in solving gender equality issues in the West yet?
\end{abstract}

Several reports and researches have pointed out to this problem. On the contrary, Islam provides a viable solution to this ever increasing problem; for Islam has a comprehensive yet simple view of gender equality. But the West, instead of understanding Islamic principles objectively, raises objections without a thorough study. However, the Western principle of gender equality has completely failed.

In this article a critical analysis of the western gender equality and Islamic principles has been carried out. It also highlights Islamic view point of gender equality. The study argues that the contemporary gender equality problems and issues that have engulfed the world particularly the Western countries can be mitigated by employing the Islamic principles of gender equality.

Key Words: gender, women, man, equality, west, Muslim, function, responsibility.

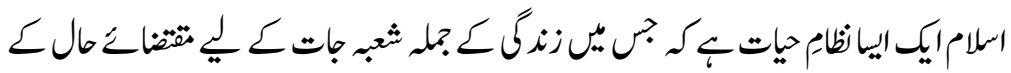

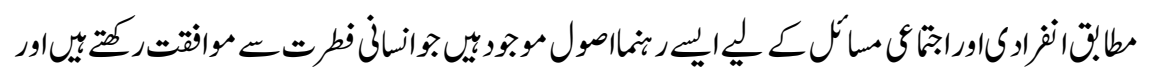

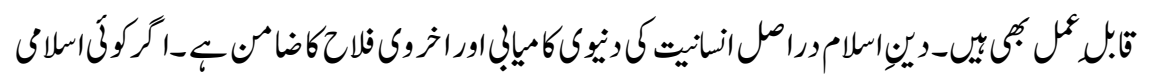

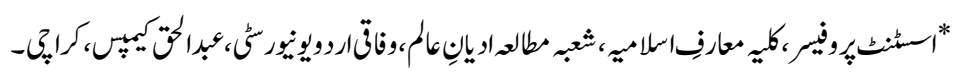




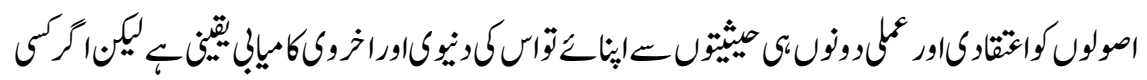

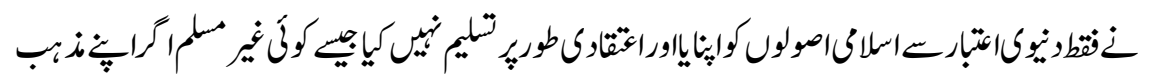

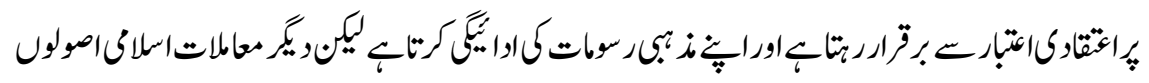

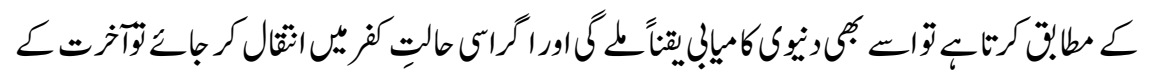

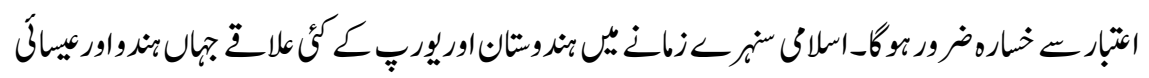

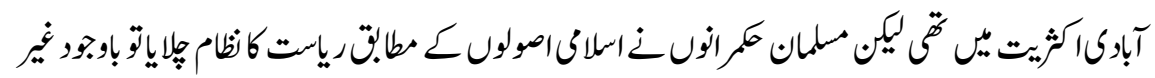

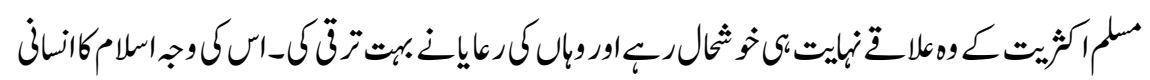

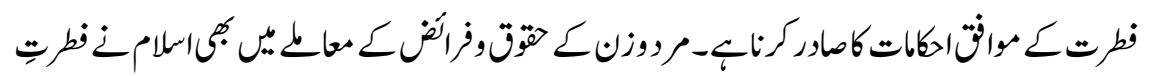

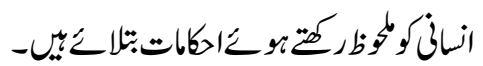

\section{اسلامكاقنقساواتكاتصور}

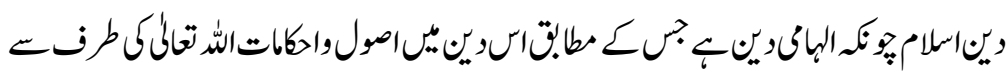

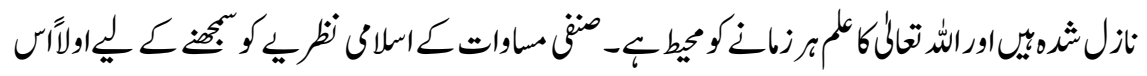

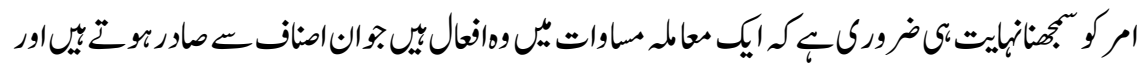

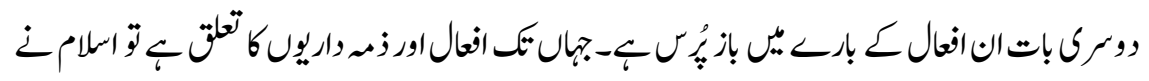

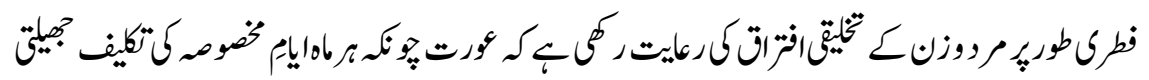

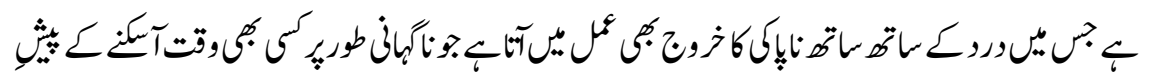

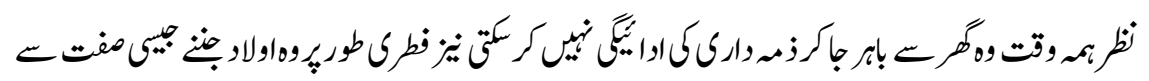

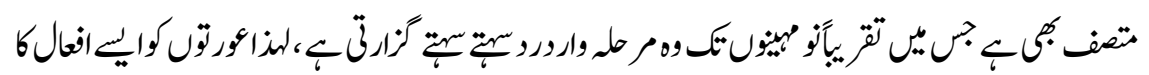

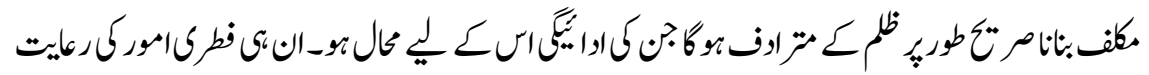

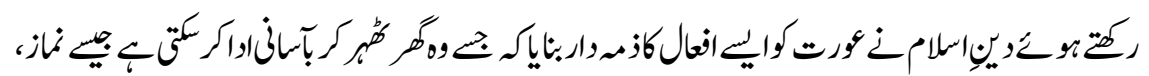

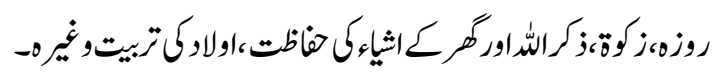

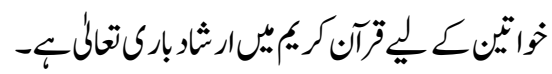




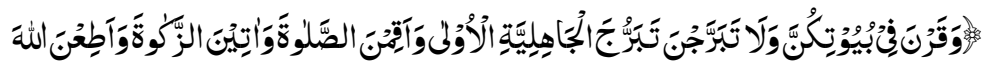

$$
1
$$

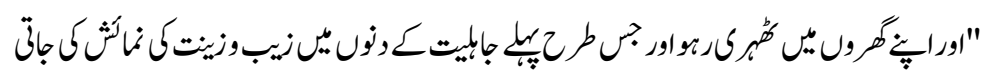

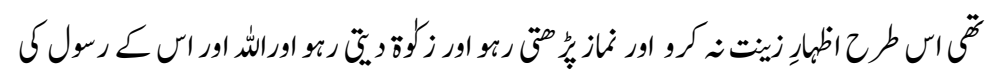

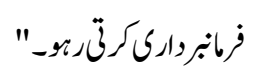

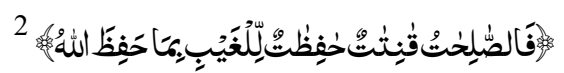

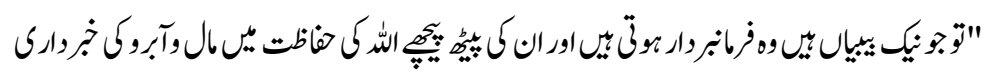

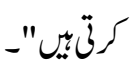

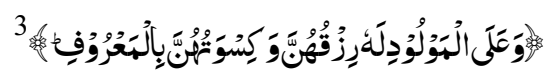

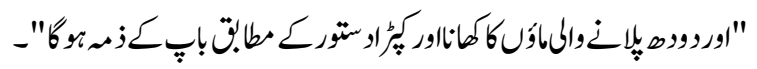

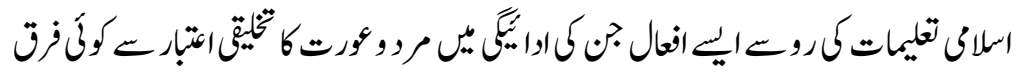

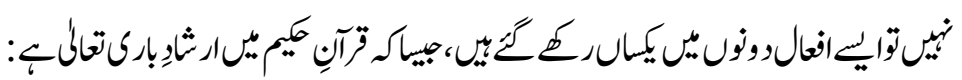

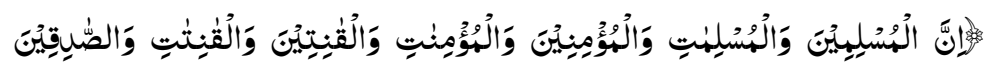

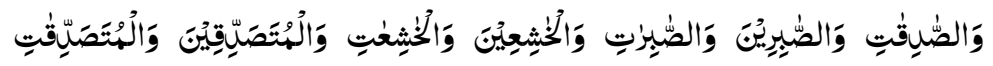

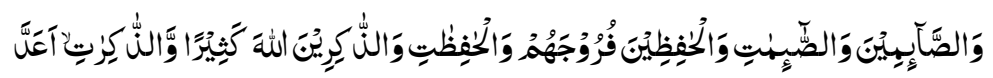

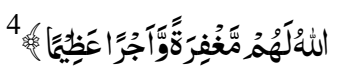

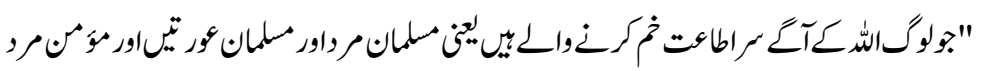

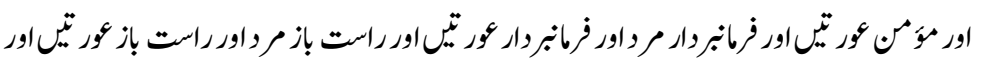

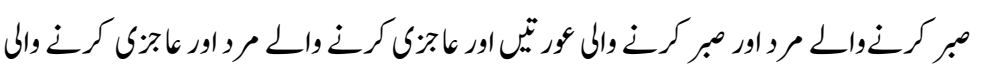

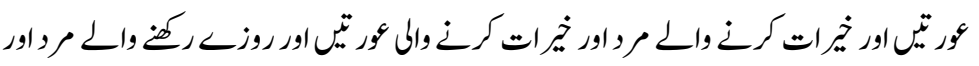

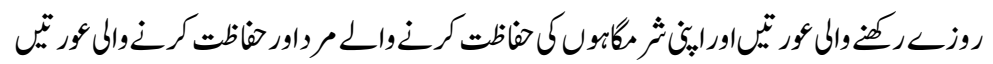

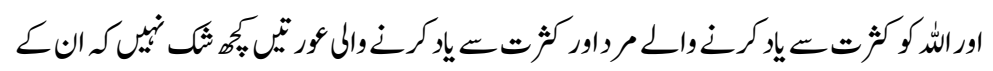

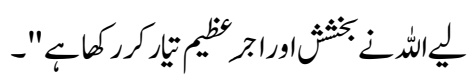




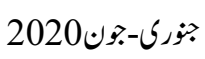

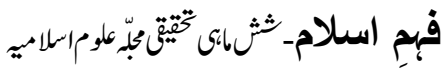

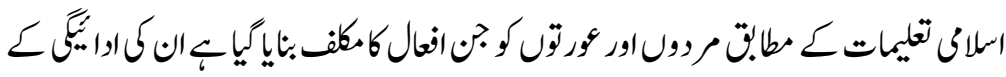

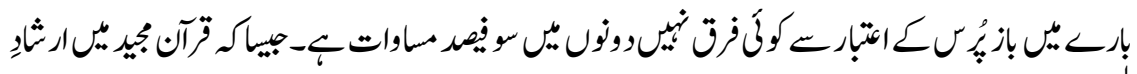

الى

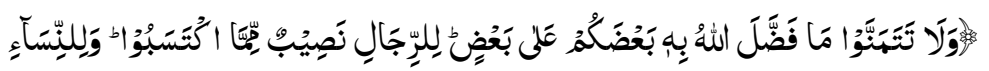

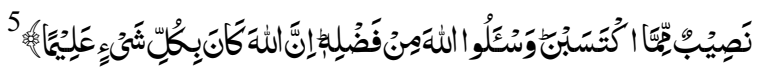

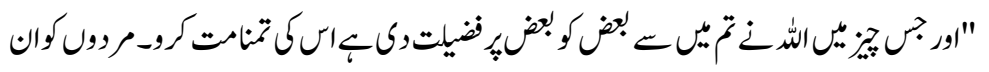

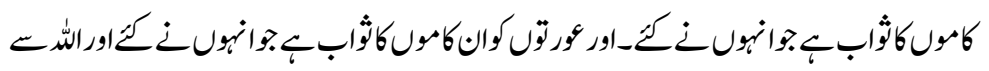

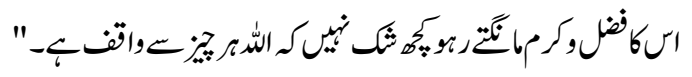

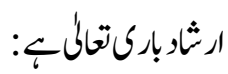

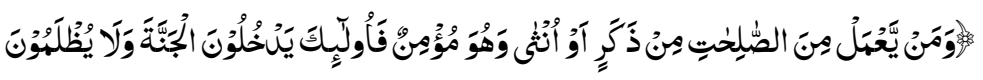

نَقِيْرَا

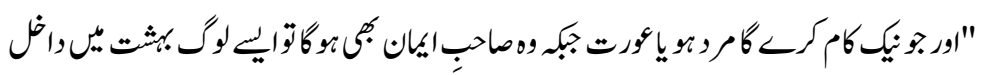

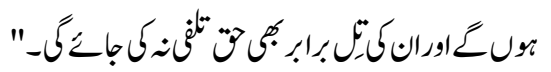

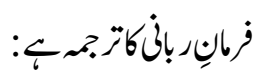

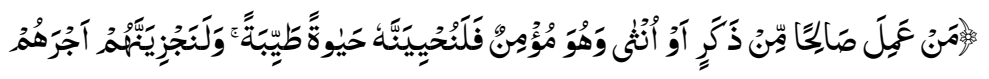

7

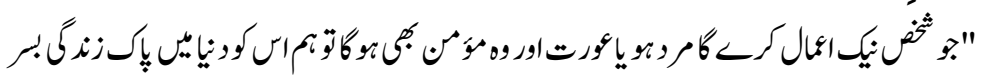

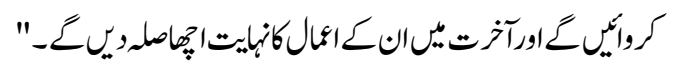

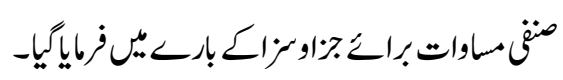

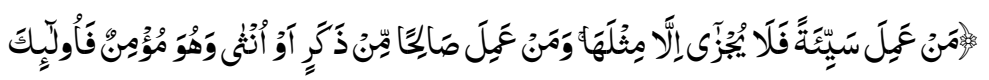

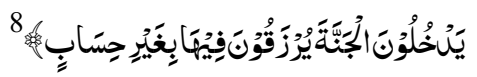

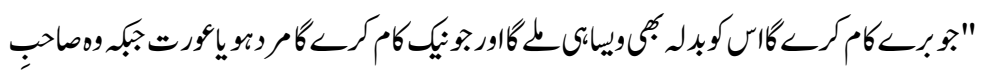

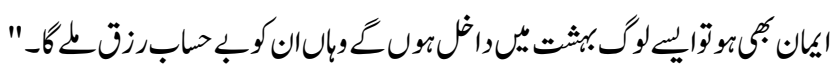

58 


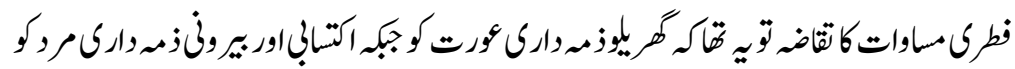

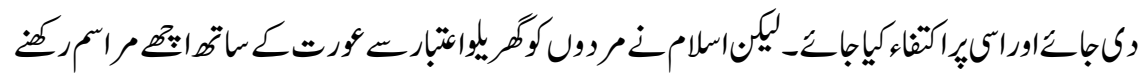

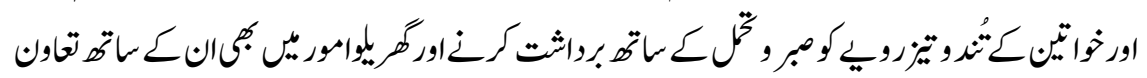

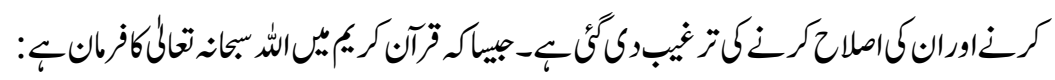

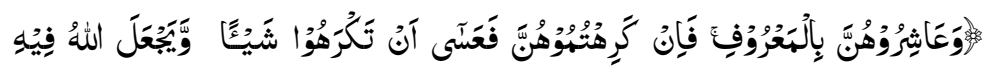
9 خَيْرَا كَثِيْرَا

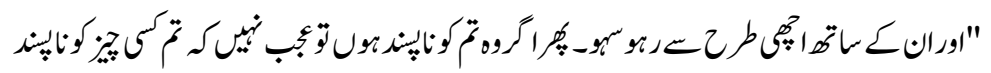

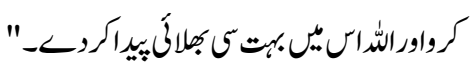

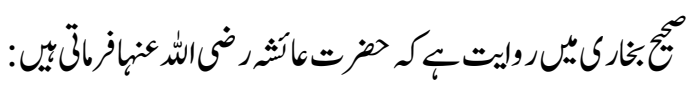

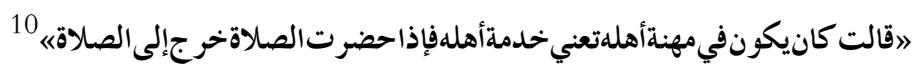

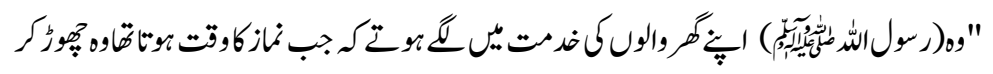
"2

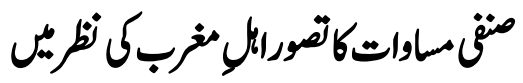

(Gender Equality)

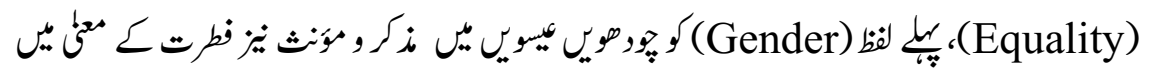

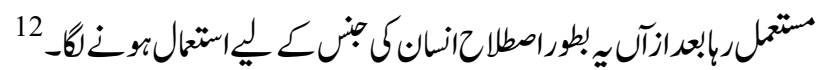

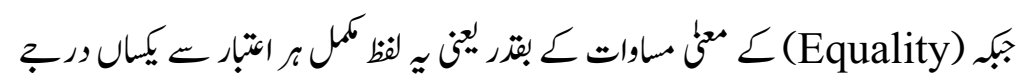

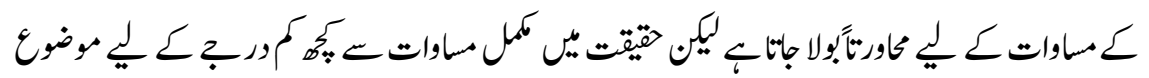

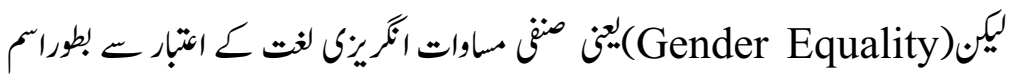

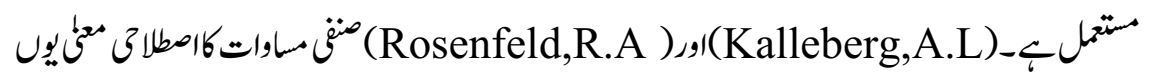
بيانكرتبن: 


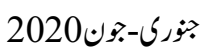

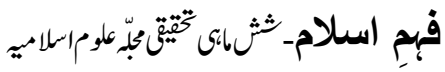

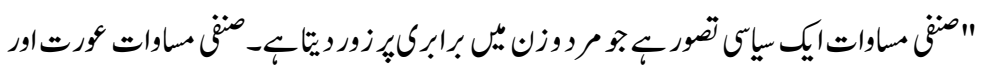

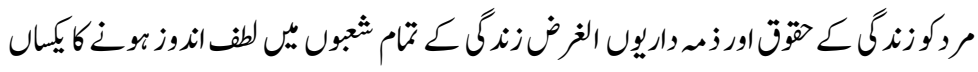

مواقع مياكرثناك-"

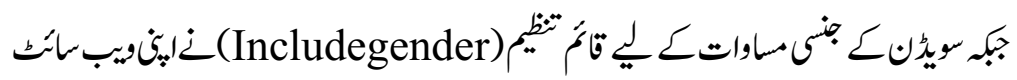

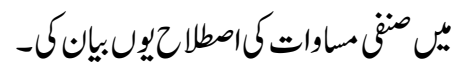

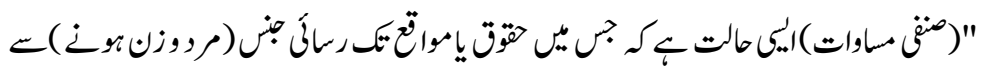

مثناثزنو_"14

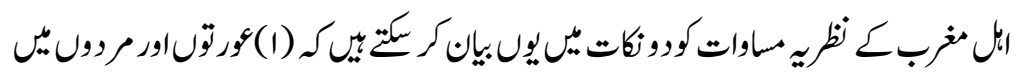

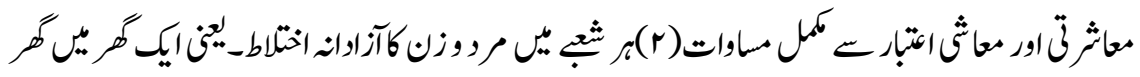

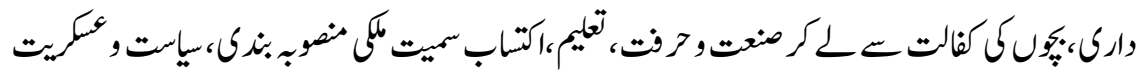

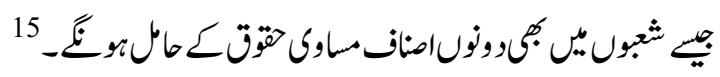

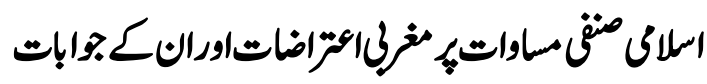

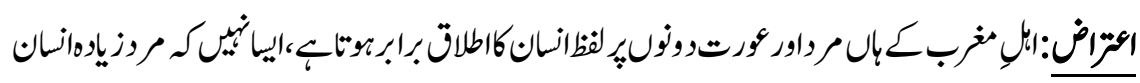

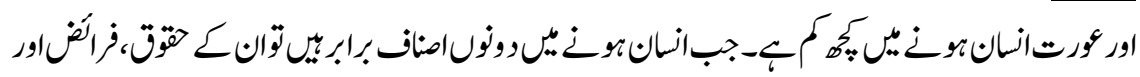

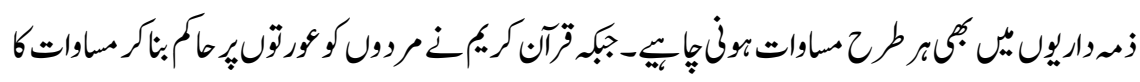

تصور بالكلى مفقوركرويا-

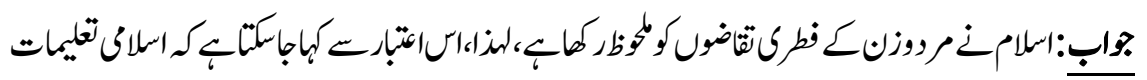

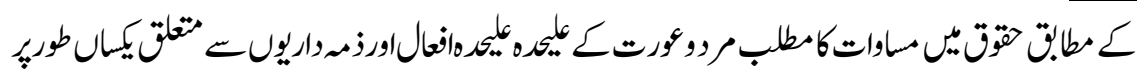

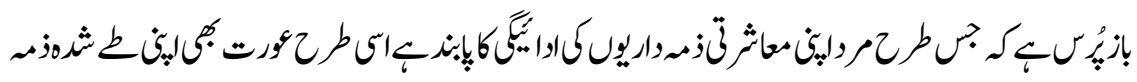

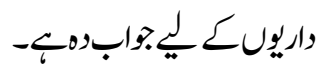

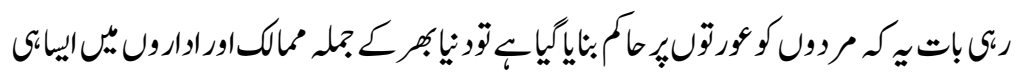

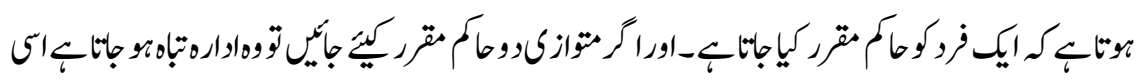

60 


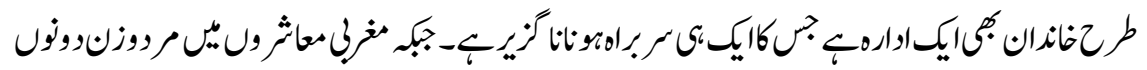

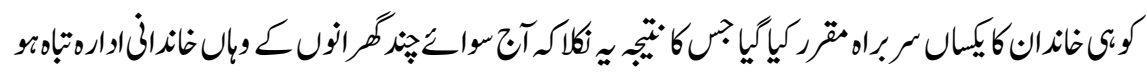

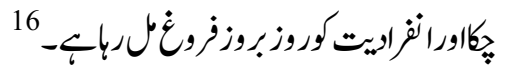

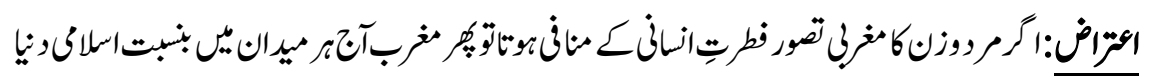

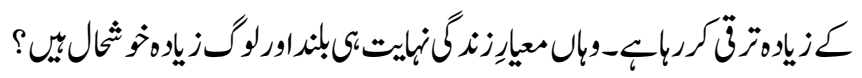

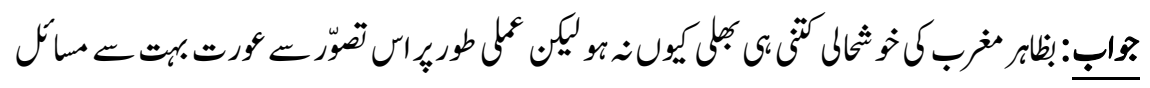

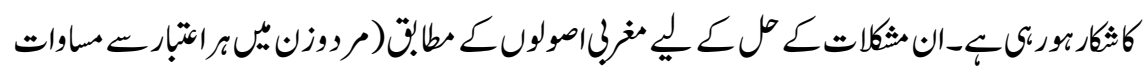

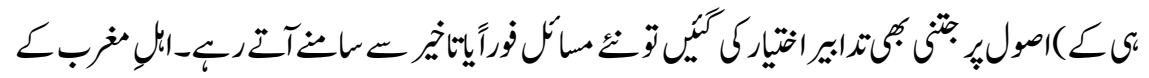

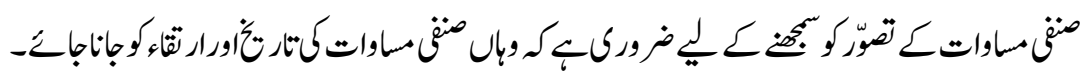

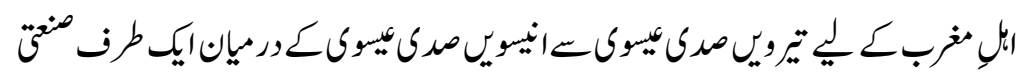

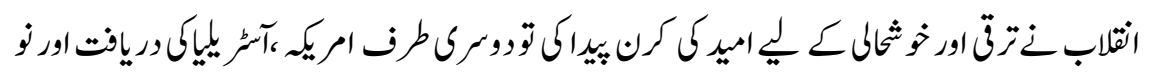

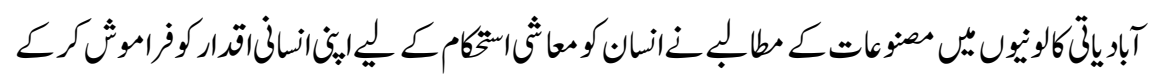

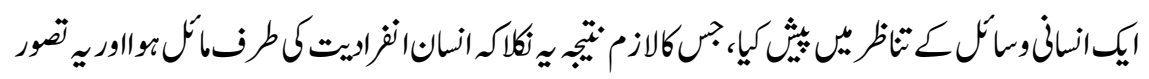

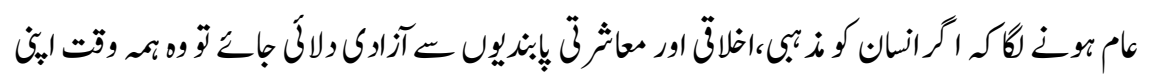

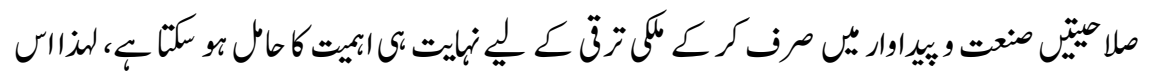

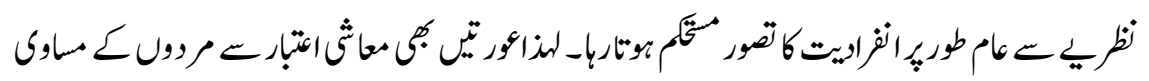

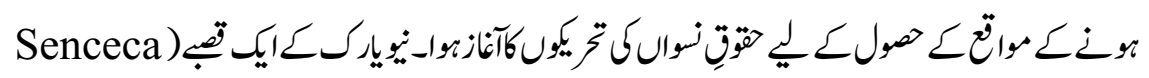
CFalls

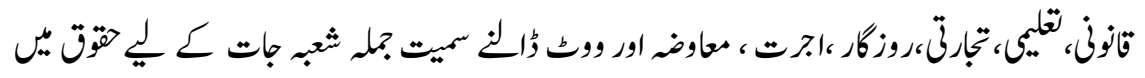

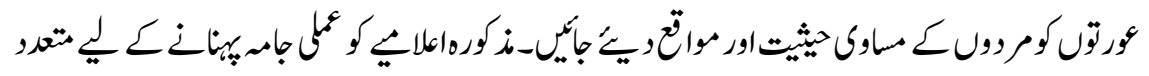

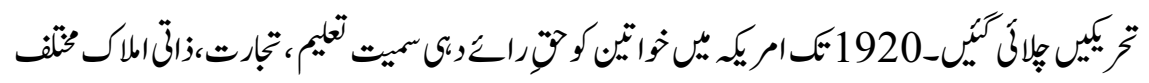

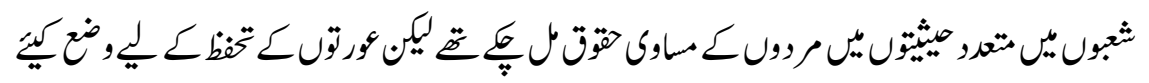

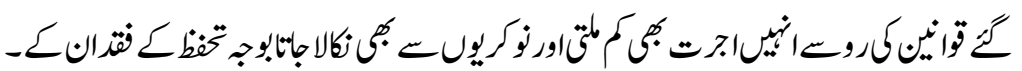




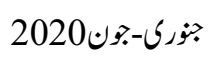

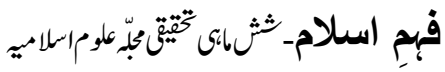

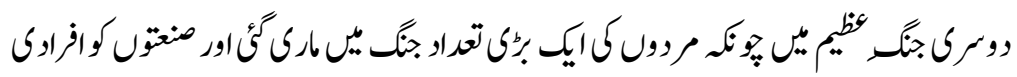

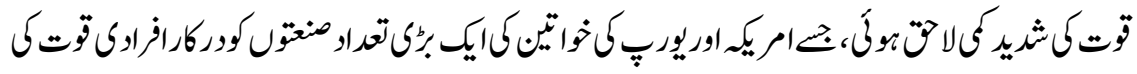

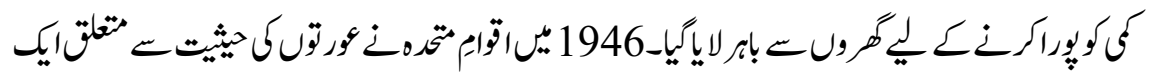

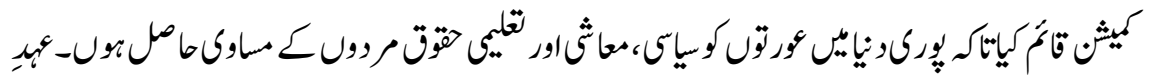

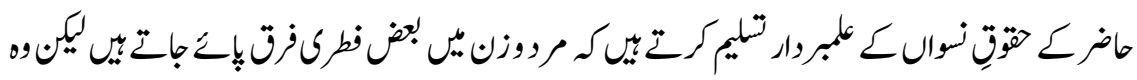

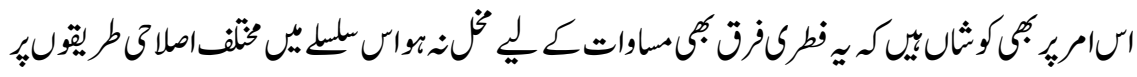

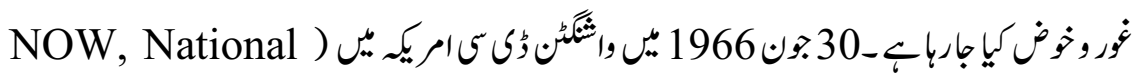

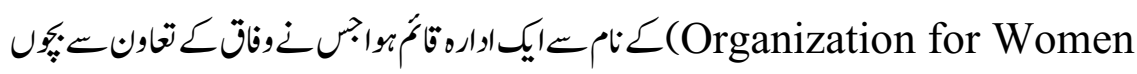

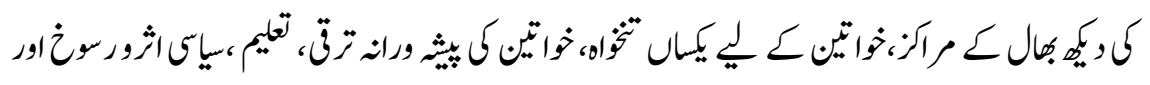

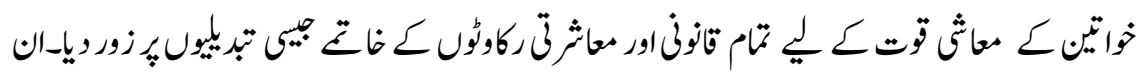

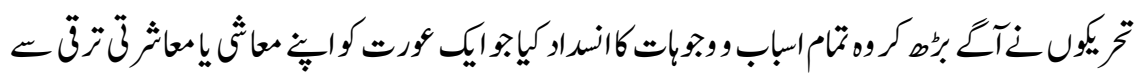

روك ستث تضش- 17

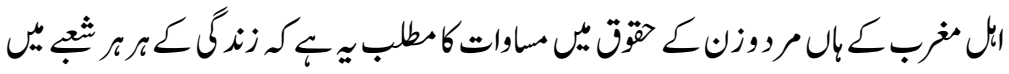

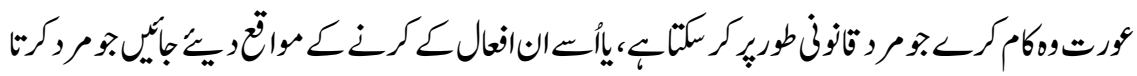

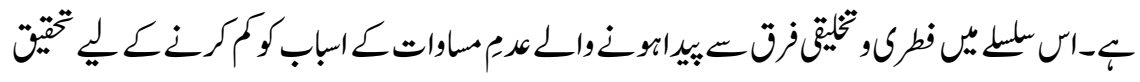

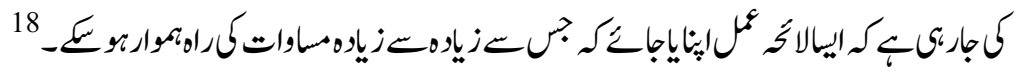

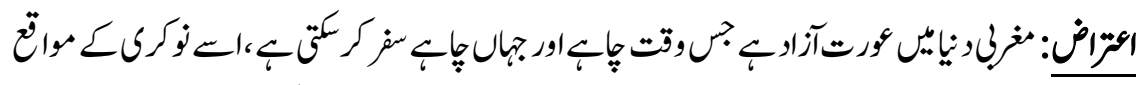

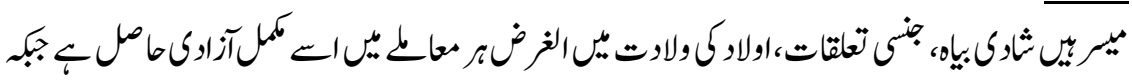

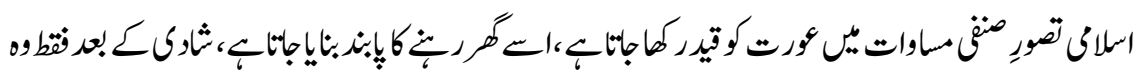

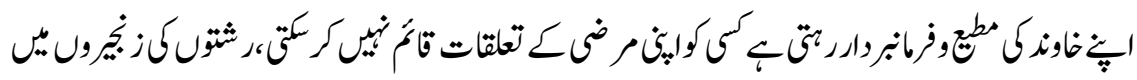

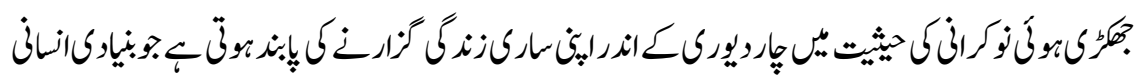

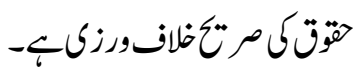

62 


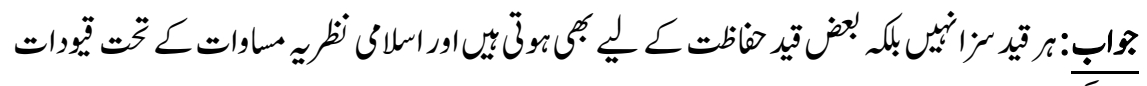

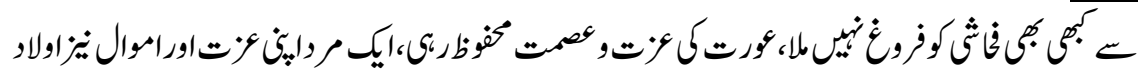

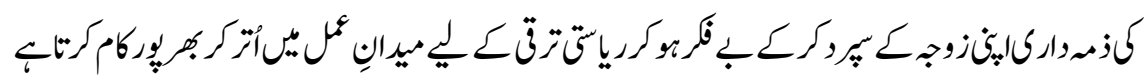

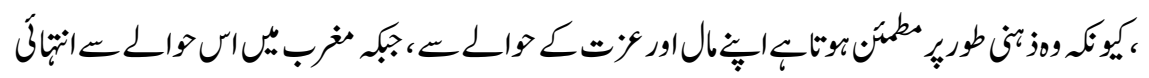

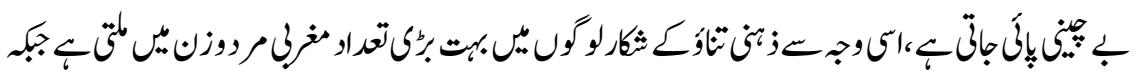

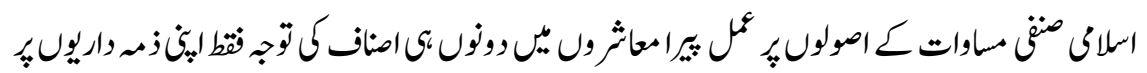

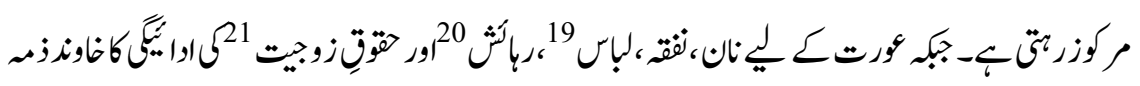

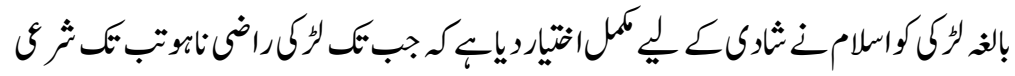

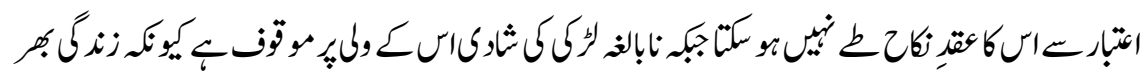

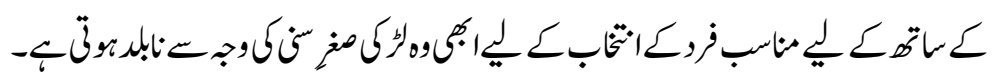

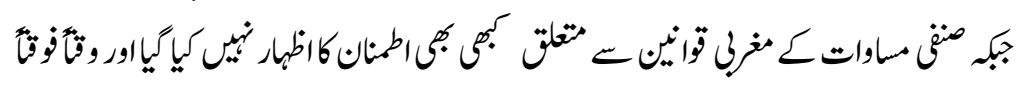

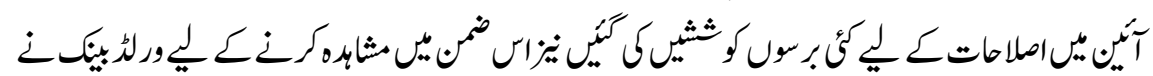

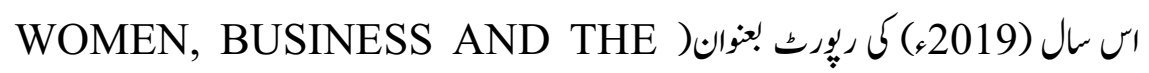

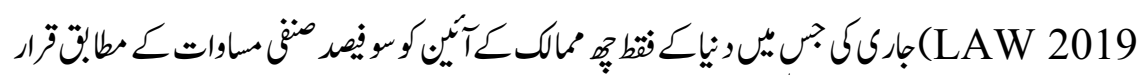

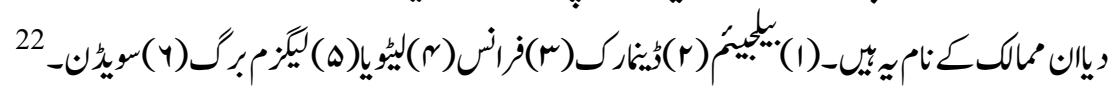

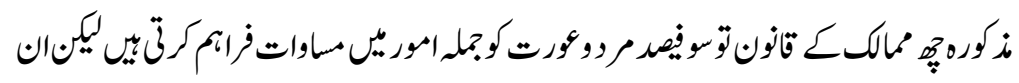

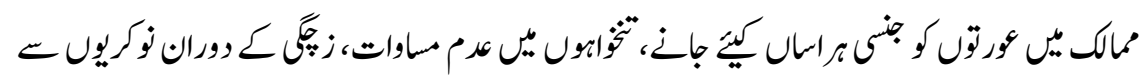

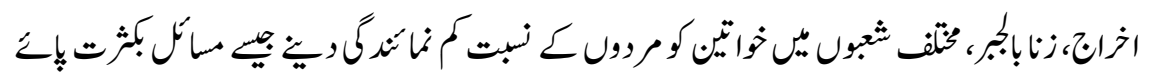

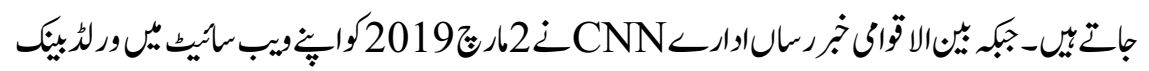

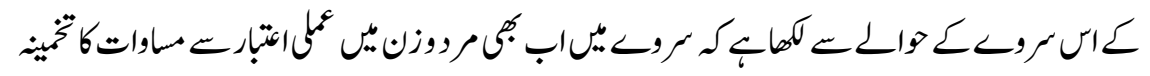

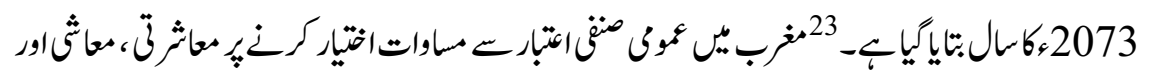

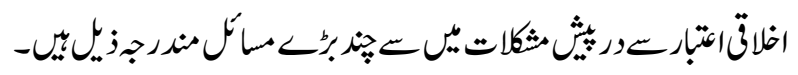




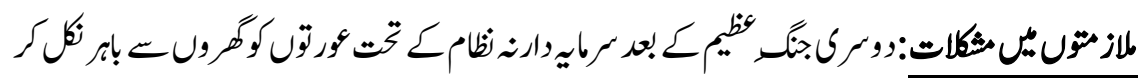

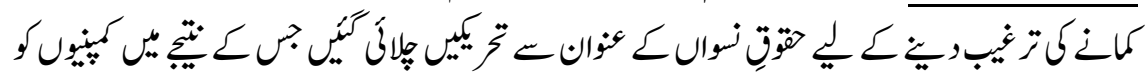

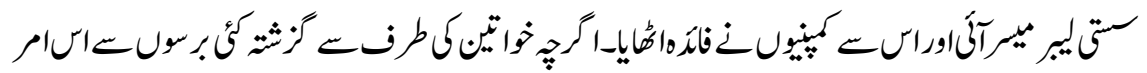

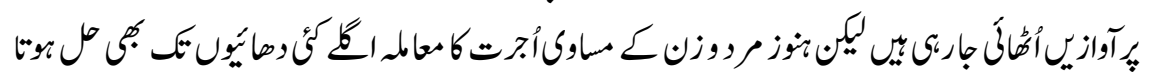

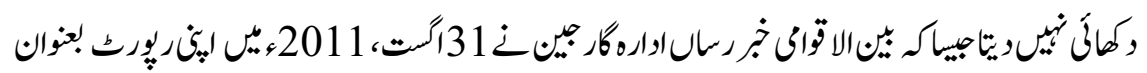
"ي انكثاف (Women executives could wait 98 years for equal pay)

"Women may have had equal voting rights since 1928, but they may have to wait another 98 years for parity in pay, research has found." 24

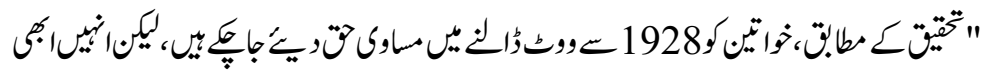

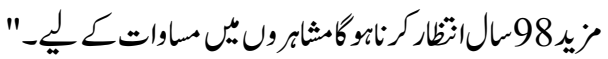

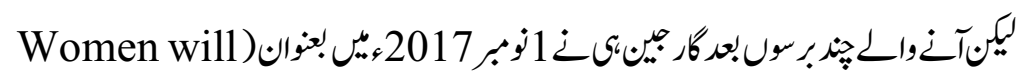
(wait 217 years for pay gap to close

"it would take 217 years for disparities in the pay and employment opportunities of men and women to end." 25

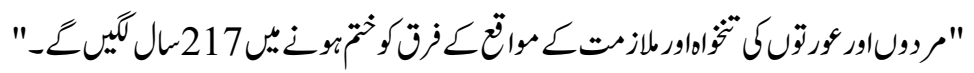

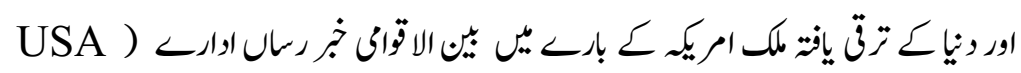

$$
\text { گre(TODAY }
$$

"WEF estimates that it will take the United States another 208 years to reach gender equality". ${ }^{26}$

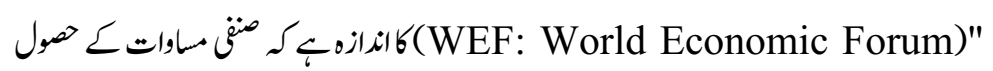
ك لِيام كيم كوزيـ208سالوركاريل-" 


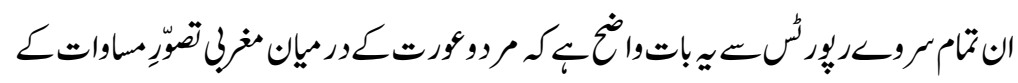

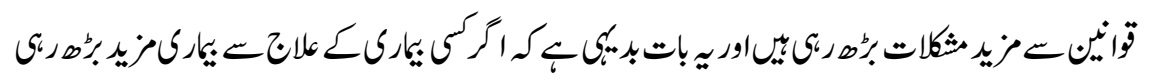

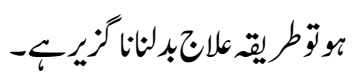

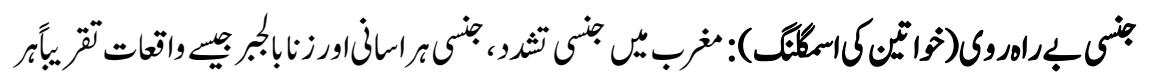

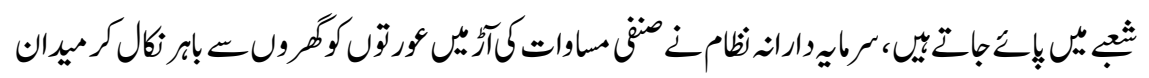

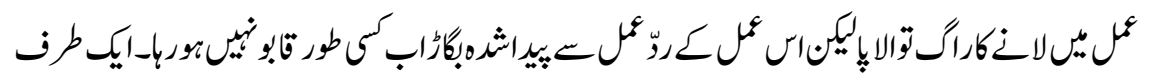

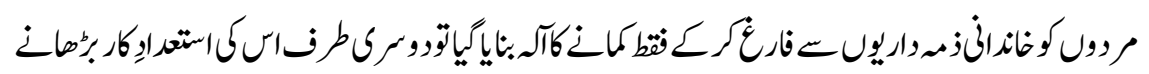

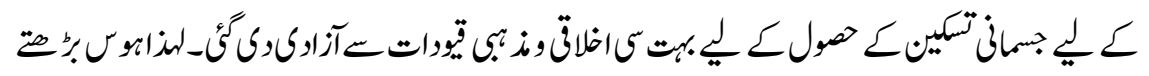

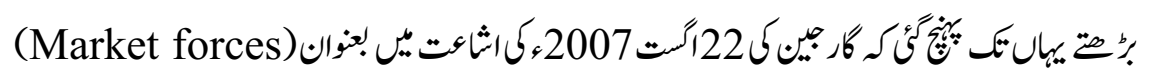

$$
\text { كزيل كنوانبية }
$$

"Many men consider buying sex as just another form of shopping, but their attitude is fuelling the trafficking of women to work in the trade"

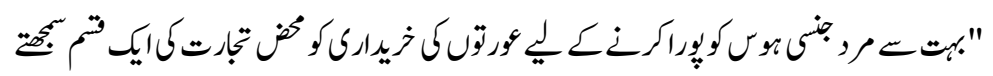

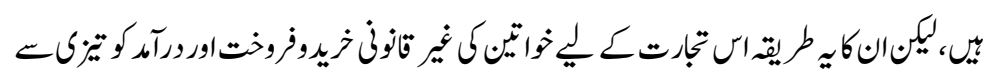
" t)

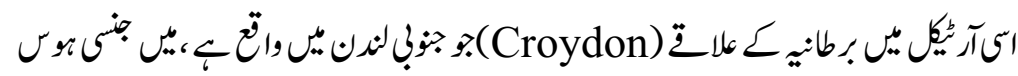

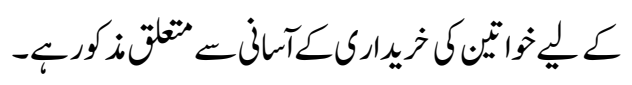

"Official figures estimate that some 4,000 women a year are trafficked into the UK to feed a growing sex industry facilitated by shrinking borders and changing attitudes towards buying sex."27 


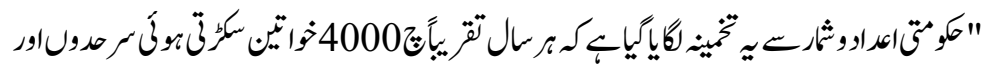

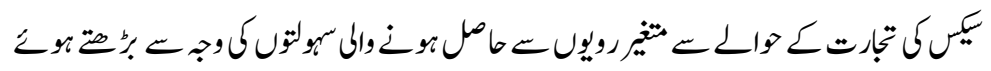

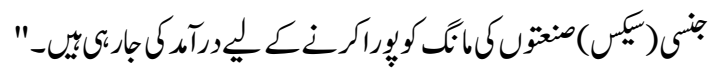

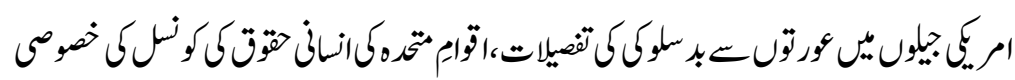

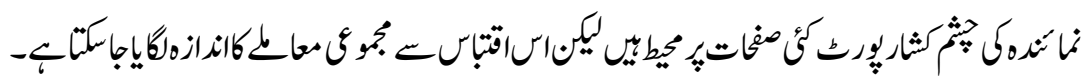

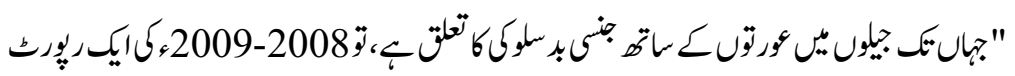

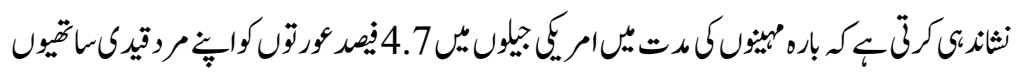

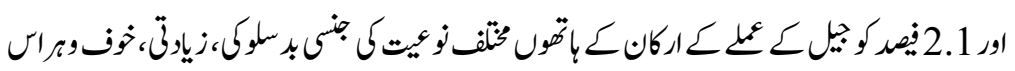

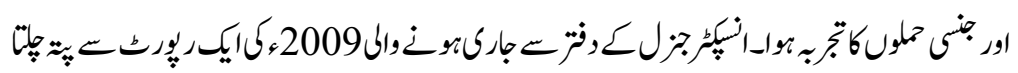

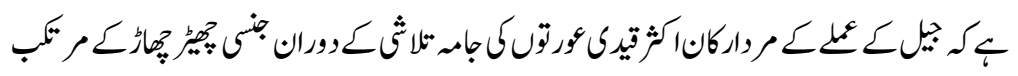

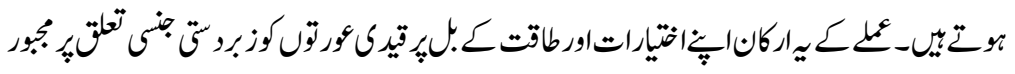
كرتبن-

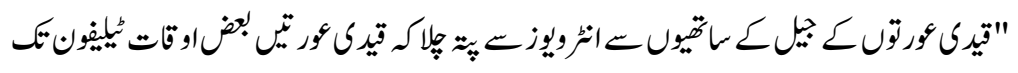

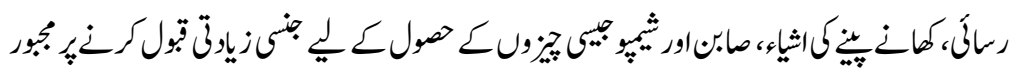

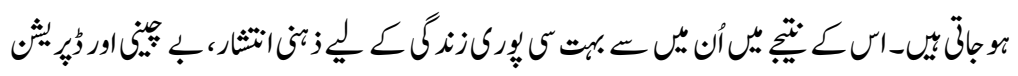

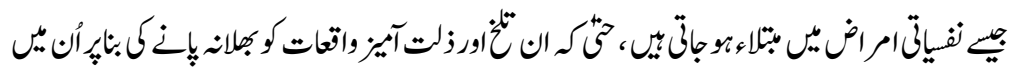

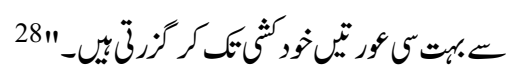

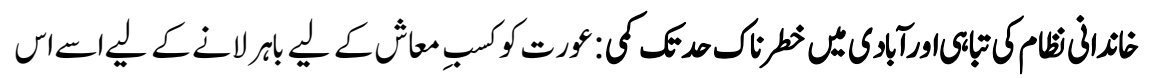

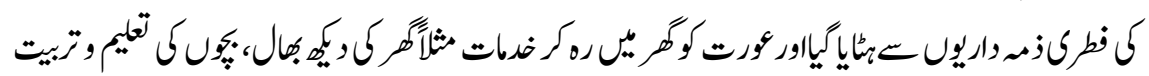

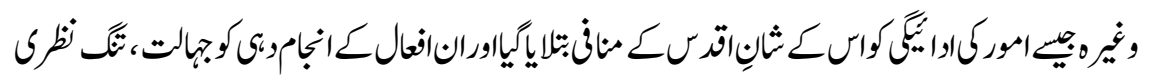

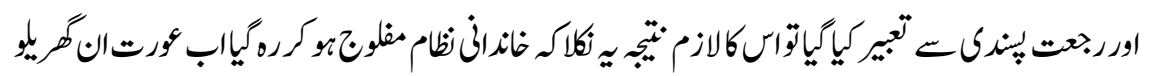

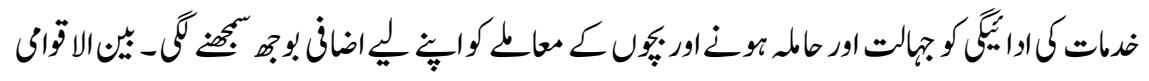

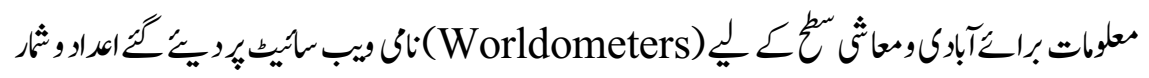




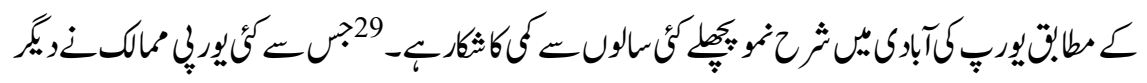

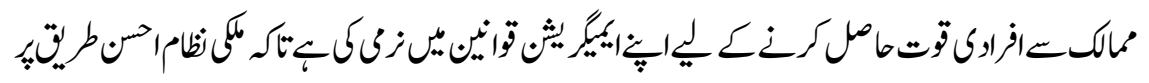

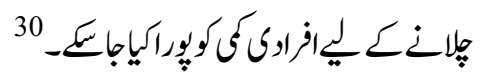

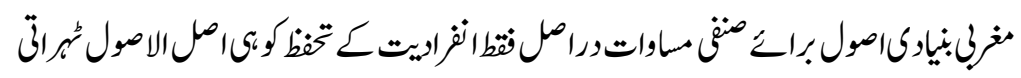

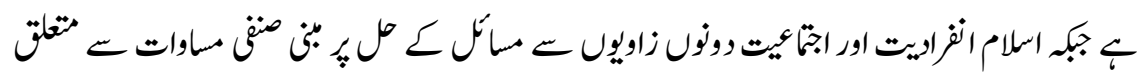

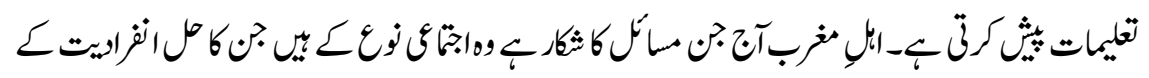

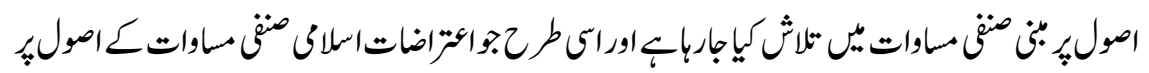

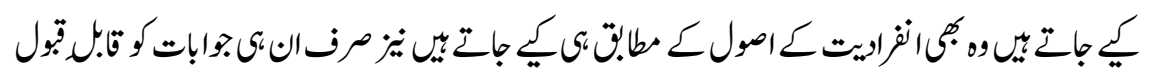

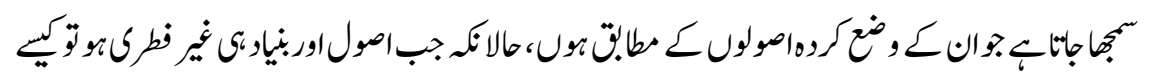

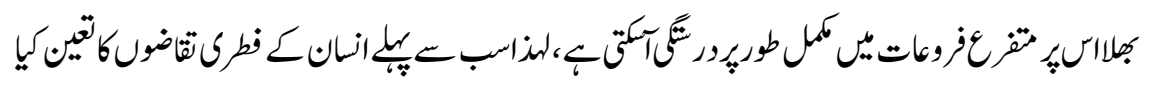

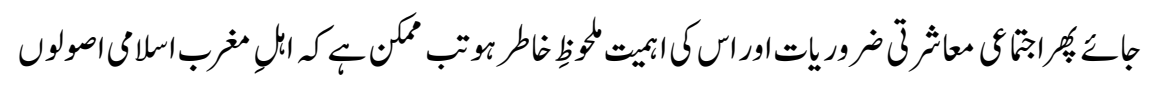

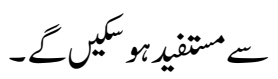

\section{فلام.كث}

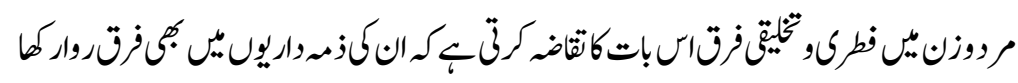

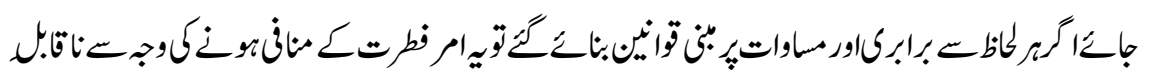

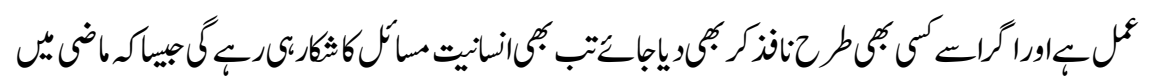

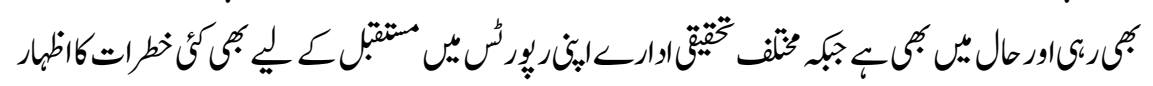

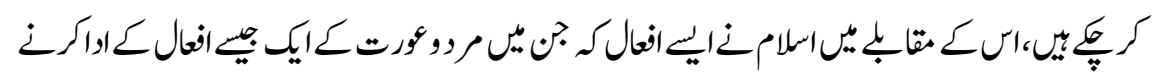

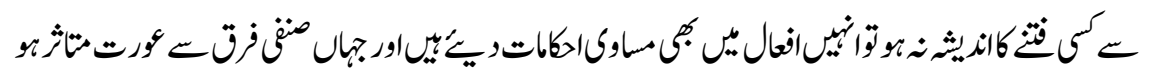

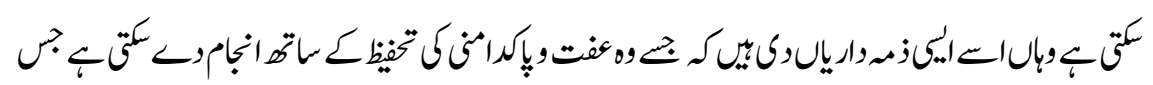

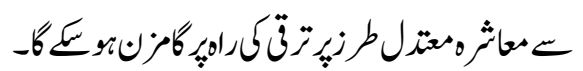

سفارثات 


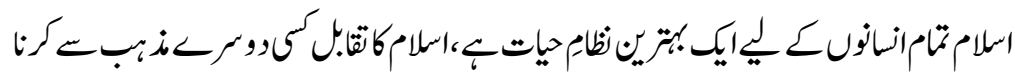

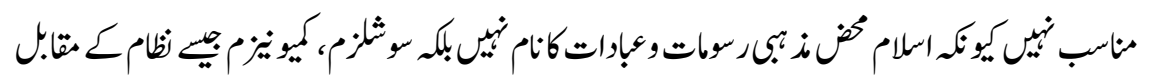

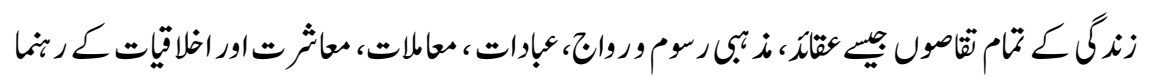

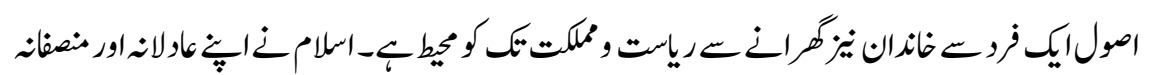

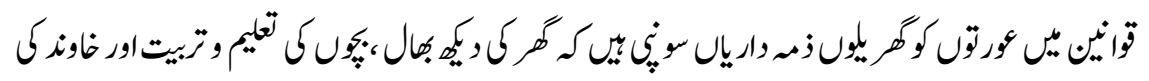

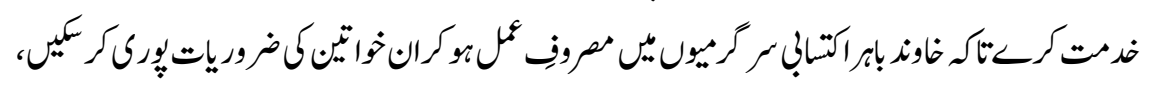

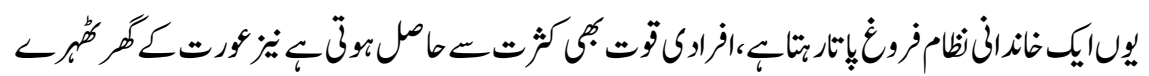

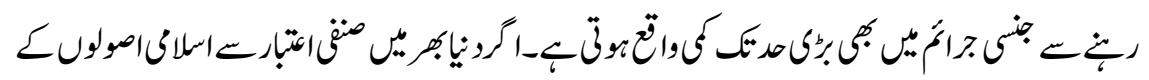

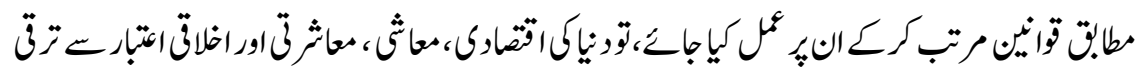

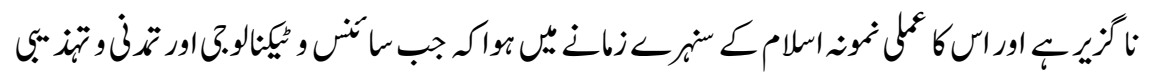

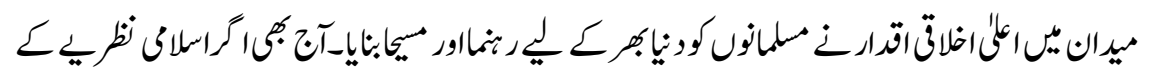

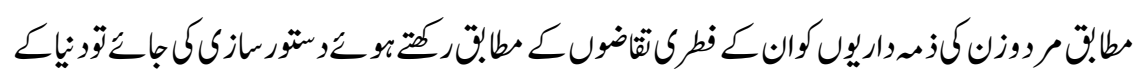

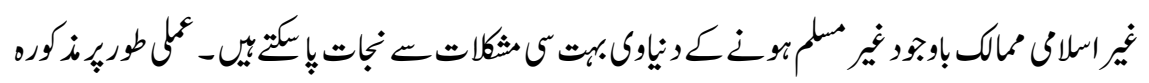

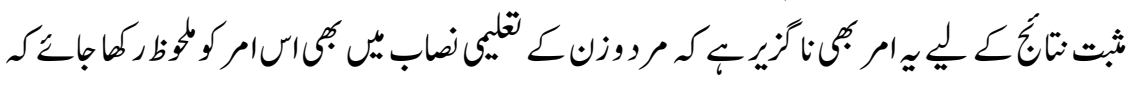

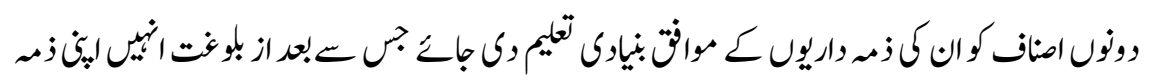

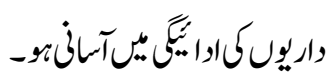

حوالد جات

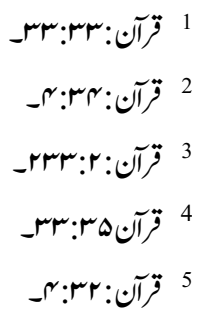




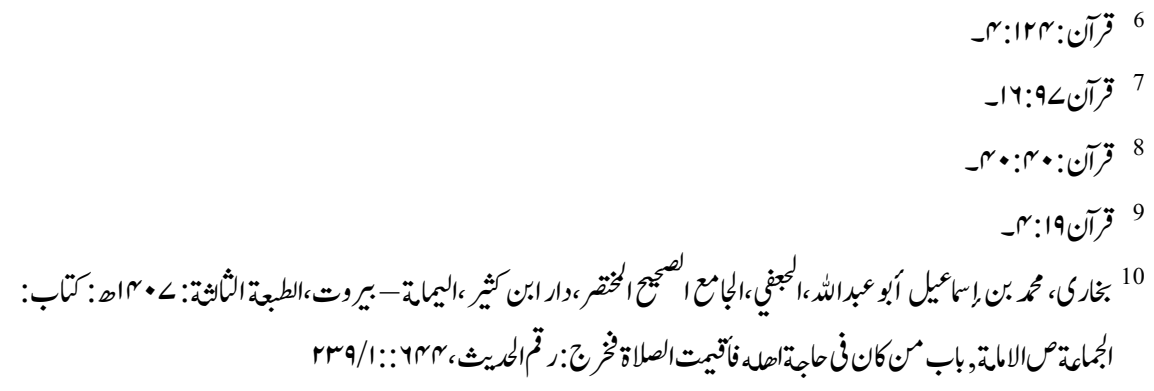

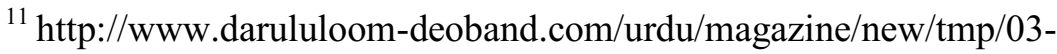
Sinf $\% 20$ Nazuk\%20Ke\%20Sath_MDU_05_May_15.htm

${ }^{12}$ The New Fowler's, Modern English Usage, R.W Burchfield, Oxford, New York, USA,2000,P\#325

${ }^{13}$ The New Fowler's, Modern English Usage,P\#257

${ }^{14}$ Rosenfeld, R. A., \& Kalleberg, A. L. (1991). Gender inequality in the labor market. A cross-national Act a Sociological perspective., 34, P\# 207-225

${ }^{15} \mathrm{https}$ //www.includegender.org/facts/gender-equality/

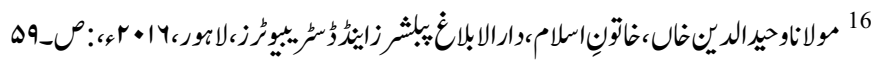

${ }^{17}$ Gender mainstreaming: Productive tensions in theory and practice, Sylvia Walby, University of Leeds,2004, P\#7

${ }^{18} \mathrm{ABC}$ of women workers' rights and gender equality, $2^{\text {nd }}$ edition, International labour office Geneva,2007,P\#12

$$
\begin{aligned}
& 19 \text { قآتسrr:r } \\
& \text { 20 } \\
& \text { 21 }
\end{aligned}
$$

${ }^{22}$ WOMEN, BUSINESS AND THE LAW 2019, A DECADE OF REFORM, World Bank, International Bank for Reconstruction and 
Development/The World Bank 1818 H Street NW, Washington DC 20433. P\#9

${ }^{23}$ https://edition.cnn.com/2019/03/02/europe/world-bank-genderequality-report-intl/index.html

${ }^{24} \mathrm{https}$ //www.theguardian.com/society/2011/aug/31/cmi-equal-pay-report

${ }^{25} \mathrm{https}: / / \mathrm{www}$. theguardian.com/society/2017/nov/01/gender-pay-gap217-years-to-close-world-economic-forum

${ }^{26} \mathrm{https}$ :/www.usatoday.com/story/opinion/2019/06/21/equality-forwomen-cant-wait-208-years-melinda-gates-column/1511613001/

${ }^{27}$ https://www.theguardian.com/society/2007/aug/22/guardiansocietysupp lement.crimel

${ }^{28} \mathrm{http}$ //magazine.mohaddis.com/shumara/229-june-2013/2566-maghribkhawateen-halt-zar

${ }^{29} \mathrm{https}$ //www.worldometers.info/world-population/europe-population/ ${ }^{30} \mathrm{https}$ //qz.com/1325640/the-european-countries-that-desperately-needmigrants-to-avoid-demographic-decline-and-those-that-dont/ 